\title{
Open Source Cellular Technologies for Cost Effective Cellular Connectivity in Rural Areas
}

\author{
Faustine Anthony \\ Department of Telecommunications and Computer Networks \\ University of Dodoma \\ Tanzania
}

\author{
Maria Gabriel \\ Department of Telecommunications and Computer Networks \\ University of Dodoma \\ Tanzania
}

\author{
Bertha Shao \\ Department of Telecommunications and Computer Networks \\ University of Dodoma \\ Tanzania
}

\begin{abstract}
Cellular coverage is often a challenge in low population density and low income rural areas of the developing world like Tanzania. This is because big Telecom companies defer from deploying their expensive infrastructure in these areas fearing operations cost. It is proposed that the use of cellular open source technology as the basis for a new rural cellular network can go a long way in meeting this challenge by providing coverage in rural areas whilst simultaneously bringing down the cost of communication. This paper provides survey of recent open source cellular technologies. It further presents a prototype based on Open BTS for cellular connectivity in developing countries. In lab performance test for the proposed prototype confirm the feasibility of deploying cellular network based on open source technologies as an alternative to conventional mobile operator networks in a bid to solve the challenge.
\end{abstract}

\section{Keywords}

SDR, Open source, Cellular technologies, OpenBTS, GSM

\section{INTRODUCTION}

Rural areas in developing countries are characterized by poor transportation infrastructure, high illiteracy levels, and scarcity of communications infrastructure [3]. The UN and ITU studies, show that when you bring communication services to an area, healthcare, economic well-being and education goes up [4]. Thus the availability of real-time voice communication in these areas is of great importance. To the large extent, the ability of residents in developing regions to access cell phone technology is limited by poor cellular coverage. Cellular network coverage is often a challenge in low population density and low- income areas of the developing and developed world [3]. This is attributed to the fact that major telecommunication companies hesitate to deploy expensive infrastructure in rural areas. Low population density without grid power and low income in rural areas, makes them unprofitable for to invest in these areas [7]. Even where cellular coverage is available the cost of making a call is considerably higher. Thus, one of the major challenges in telecommunications today is to provide quality and efficient cellular communication services in rural areas at low cost.

Taking Tanzania as an example, mobile penetration in urban areas is more than $80 \%$ while in rural areas it is only $25 \%$. To address this challenges, the Tanzanian government has taken an interest in finding a solution by setting up The Universal Communication Access Fund (UCAF) aiming at providing low-cost telecommunication services in rural and underserved areas [1]. The fund was established in 2006 with initial 48 billion to provide low- cost telecommunication connections in these areas across the country where 1.6 million people were targeted in the first phase of the programme. Despite this government initiative, the challenge of coverage in rural areas has not been solved.TCRA statistics show that over 5 million people have no access to communications. As a result, the problem of cellular coverage in rural areas cannot be completely solved by traditional cellular technologies and conventional telecommunication companies which are driven by the need to make a profit.

On the other hand the advancement of Software Defined Radio concepts has led to the very rapid and steady development of cellular open source technologies that are free to use, modify and implement [12][10]. The open source cellular technologies applies to both software and hardware in which essential design information, such as schematics, firmware, or driver source code, is made openly available. The Universal Software Radio Peripheral (USRP) is a well known example of the open source development model applied to a hardware product. USRP boards are developed by Matt Ettus ${ }^{1}$ mostly for the users of GNU radio ${ }^{2}$ which is an open-source software development toolkit [14].

Open source cellular technologies implement most of the cellular networks functions using a software in one device. These technolo-

\footnotetext{
${ }^{1}$ The world's leading supplier of software defined radio platforms, including the Universal Software Radio Peripheral (USRP) family of products(https://www.ettus.com/)

${ }^{2}$ Open-source software development toolkit that provides signal processing blocks to implement software radios(http://gnuradio.org/)
} 
gies can be used to address the rural coverage challenge by enabling the design of newer, cheaper and simpler cellular networks. Opensource cellular technologies have the potential to create a wealth of significant opportunities, such as reducing the investment and operational cost of the traditional cellular network while creating cost-effective services for use by a rural user in developing countries.

In recent years, open source implementations of various GSM components have been introduced as viable candidates for cellular system testing, research, and deployment [13]. This paper focus on current developments regarding open source cellular technologies. Section II, provide a brief overview of open source cellular technologies. In Section III, implementation and performance testing of openBTS based cellular-network in lab setting is presented. Finally, conclusion, recommendation and the future works are discussed in Section IV.

\section{OPEN SOURCE CELLULAR TECHNOLOGIES}

Cellular open source technologies implement cellular networks using open source software technologies (running on normal PCs). They use Software defined radio (SDR), a core technology for future-generation wireless communications. Traditionally, the communication physical layer functions are implemented in hardware. This implies a slight modification in any one of the function, will require redesigning and implementing a new hardware. The hardware redesign and implementation is costly and time consuming [14].

SDR configure a radio platform like a freely programmable computer using an appropriate programming interface. It aims at implementing some or all of the physical layer functions such as air interfaces and signal processing blocks using software in one device. Having these functions implemented in software, they become flexible, reconfigurable and saves prototyping time.

SDR provides an efficient and comparatively inexpensive solution to the challenge of building multi-mode, multi-band, multifunctional wireless devices that can be adapted, updated, or enhanced by using software upgrades [9][6].

SDR technologies emphasize on software implementation of the traditional tasks of telecommunications such as modulation, demodulation, filtering, multiplexing, de-multiplexing and others, as it is cheaper than a hardware implementation. Several popular projects have emerged that offer varying network architectures and allow users to implement a cellular network in different capacities depending on individual needs. These includes OpenBTS, OpenBSC, OpenLTE and YateBTS.

OpenBTS $3^{3}$ this is the first free software implementation of the industry-standard GSM protocol stack and is the only open source solutions for GSM. It provides network functions like GSM registrations, location updating, handover and mobility management [8]. It is a Linux application that uses SDR to present a standard 3 GPP air interface to user devices. It uses the GSM air interface on the radio side which makes it compatible with most $2 \mathrm{G}$ and $3 \mathrm{G}$ handsets. On the backend, it uses the open source PBX implementation which considers mobile phones as SIP clients [8].The PBX

\footnotetext{
${ }^{3}$ OpenBTS: http://openbts.org/
}

can be implemented by either Asterisk ${ }^{4}$. Free Switch ${ }^{5}$, or Yate ${ }^{6}$ open source VOIP software. For the OpenBTS's air interface, multiple hardware options are supported with variants from the USRP product line being the most commonly used [13].

Recently OpenBTS has announced the public release of OpenBTSUMTS 1.0, providing data capability for $3 \mathrm{G}$ networks. The architecture collapses GSM and 3G into a single platform, thus simplifying the core network and enabling web services directly to mobile handsets [2]. The main advantage of OpenBTS is the low cost of installation and it is compatible with most of the handsets that are already in the market. Another advantage is that OpenBTS allows bug fixing over the air or another remote reprogramming thus reducing both time and cost associated with operation and maintenance [5]. Furthermore, it can work and service under extreme conditions this is particularly important if it is to be used in off-thegrid rural and disadvantageous areas where the infrastructure and backhaul are not efficient. OpenBTS can be used as an alternative to the cellular network in post-disaster situations [15][11]

OpenBSC 7 was originally developed as a research and experimentation platform. It implement the Abis protocol, as implemented in GSM Base Station Controller (BSC). The key difference between the OpenBSC project and the OpenBTS project is the interface that they use to communicate with the user equipment (UE). OpenBTS implements the Um interface whereas the OpenBSC project implements the Abis interface.

The main advantage of OpenBSC is that it can be used in place of a proprietary BSC within an existing GSM network, or simply combined with an off-the-shelf BTS and configured to provide a turnkey (stand-alone) network. Thus it is possible to bypass the traditional components such as MSC, HLR, and VLR. Also OpenBSC supports well some extended features like SMS messaging and mobility management as it uses the E1 channels. The use of commercial BTS allows for the operation of a scalable multi-cellular network. Additionally the lower layers of the OpenBTS stack may be configured to interface to OpenBSC in a traditional network architecture [13]. The main drawback as far as OpenBSC is concerned is the requirement of a commercial BTS, which drastically increases the overall cost and thus the initial investment for an OpenBSC project is high. Apart from that OpenBSC only support few models.

YateBTS ${ }^{8}$ is an open source GSM Base Station software used to create a $2 \mathrm{G}$ GSM network, either standalone or connected to the public telephone network. It is designed to be compatible with both 2.5G SS7/MAP and 4G IMS core networks. YateBTS is built by borrowing some functionality from OpenBTS. It runs on SatSite 9 , a $2.5 \mathrm{G} / 4 \mathrm{G}$ base station designed for areas with limited infrastructure. YateBTS can be used by mobile network operator labs, Machine to Machine(M2M) application development, mobile phone vendors, academics and security researchers.

\footnotetext{
${ }^{4}$ Asterisk: standard open-source PBX solution (http://www.asterisk.org/)

${ }^{5}$ Free switch: a full featured PBX capable of routing both Voice and SMS traffic (https://freeswitch.org/)

${ }^{6}$ Yate: advanced, mature, flexible telephony server that is used for VoIP and fixed networks (http://www.yate.ro/)

${ }^{7}$ OpenBSC: (http://openbsc.osmocom.org/trac/wiki/OpenBSC)

${ }^{8}$ YateBTS: (http://yatebts.com/)

${ }^{9} \mathrm{http}: / /$ yatebts.com/sat_site.php
} 
Open eNodeB ${ }^{10}$ is a soft $4 \mathrm{G}$ LTE base station which is implemented using a standard PC and a low cost software radio front end. The entire physical layer, data link layer and network layer processing is done in real time inside the PC, so no dedicated Long Term Evolution (LTE) hardware is necessary. Since it simulates an LTE infrastructure it can work without a real LTE network behind it. It also possible to use Open eNode short, it is possible to use open eNodeB as a Wi-Fi access point.

Open eNodeB replaces the expensive hardware that is connected to the mobile phone network that communicates directly with UEs. Currently, the potential use of Open eNodeB is limited to being a low cost LTE network simulator. Since it is SDR implementation, it is very easy to add and test new LTE features. The flexibility of adding and decreasing modules, makes Open eNodeB ideal for LTE simulation and test-bed. It is expected that in the near future Open eNodeB can act as the basis of a low cost LTE networks especially in rural and disadvantageous regions. The real challenge of Open eNodeB is that $4 \mathrm{G}$ has much higher bandwidth requirements than $3 \mathrm{G}$, thus building it in the power limited environment of rural and disadvantageous areas is tricky.

\section{PROTOTYPE IMPLEMENTATION}

The prototype was implemented using OpenBTS technologies. Figure 1 shows the network setup for the experiments. The four main components of the architecture are; GNU Radio, USRP, OpenBTS and Asterisk. GNU Radio provides SDR signal processing blocks while USRP provides external RF hardware to GNU Radio. The USRP and GNU Radio implement the physical layer and are responsible for modulation and synchronization. Asterisk act as a database for subscribers and call switching server and OpenBTS provides the GSM stack. The Control function is located midway between GSM stack and the SIP server. It interprets the commands from GSM stack (Downlink) or from SIP (Uplink) and executes the corresponding functions. Figure 2 shows the OpenBTS core modules.

The prototype network performs optimally when it is operated in a free and unused channel. However, since OpenBTS is constrained to operate in the GSM $900 \mathrm{MHz}$ band which is fully occupied by commercial operators it was impossible to get a free and unused channel in this band. The prototype therefore was used and tested amidst interference from other sources which reduces network performance. The lack of pre-specified and reserved research channels in the GSM band in Tanzania adds to the problem. The only possible solution to this was to scan for a dark spot where external signals are weak or relocating to a rural area where there are no or only few commercial operators service is running.

Also, the prototype requires each new subscriber to be manually registered. This was done by obtaining subscriber's International Mobile Subscriber Identity (IMSI) and add it to the configuration files. This approach is not an efficient and practical method for adding new subscribers to cellular network.

Additionally, the internal clock that ships with the USRP did not meet the synchronization requirements of GSM standard. The GSM standard requires a clock efficiency of at least 0.1 parts per million (ppm). As the results it was not possible to use $1800 \mathrm{MHz}$ GSM band (since drift is higher at upper frequencies). This challenge caused some handsets fail to be registered on the GSM network.

\footnotetext{
${ }^{10} \mathrm{http}: / /$ bellard.org/lte/
}

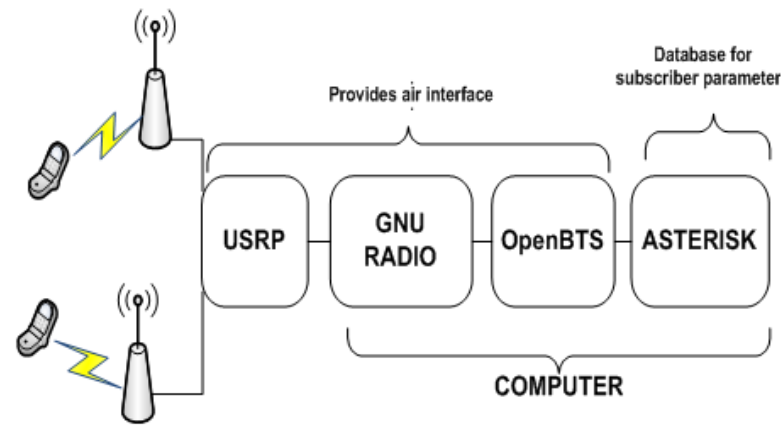

Fig. 1. Prototype System Architecture

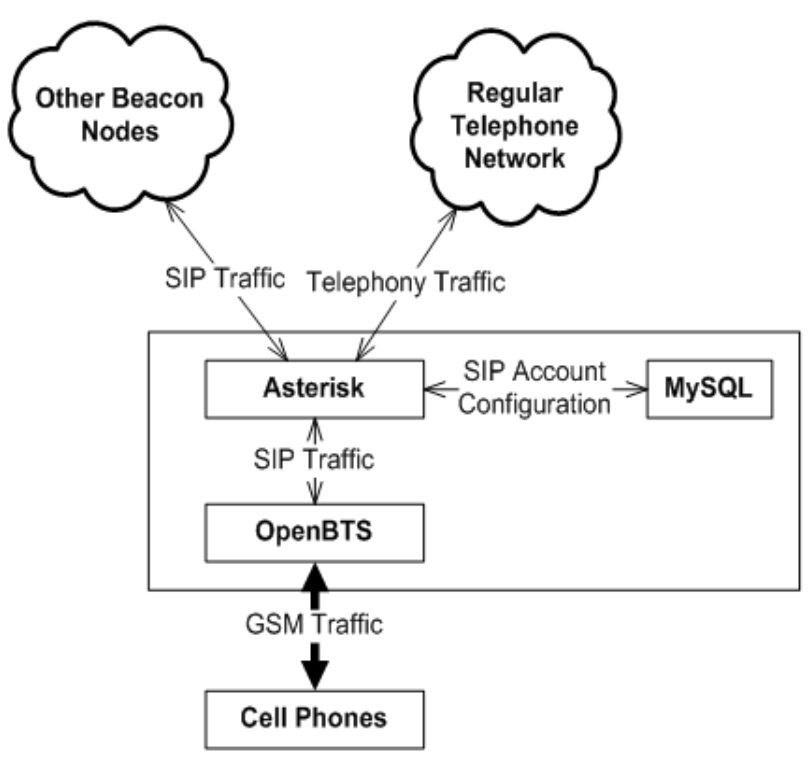

Fig. 2. OpenBTS Core Modules [10]

\section{RESULTS AND DISCUSSION}

After successfully implementation of a cellular network prototype, the next step was to test and analyse the performance of the prototype. The testing was conducted with the aim of obtaining the maximum range of coverage of the network and variation of incall parameters with respect to changes in distance of separation. USRP and the host computer were stationed at a fixed point, which was also the location of a stationary caller A, while another user (caller B) initiated calls at different distances from caller A. For the testing of variation in-call parameters which include Uplink frame erasure rate (UPFER) in \%, Uplink Received Signal Strength Indication (RSSI) in dB, Downlink Received Signal Strength Indication (RSSI) in $\mathrm{dBm}$ and Downlink bit error rate in \% with distance the system was set as in Figure 2 For testing purpose the parameters of Table 1 were chosen.

Figure 3 shows the variation of uplink RSSI Indication at BTS with distance. It can be seen that the uplink RSSI decreases as the distance of separation from MS to BTS increases.

The variation of downlink RSSI from MS with distance is shown in Figure 4, where values of downlink RSSI was taken at different 
Table 1. Constant Parameters during Testing.

\begin{tabular}{ll}
\hline Parameter & Value \\
\hline Frequency of operation & $900 \mathrm{MHz}$ \\
Transmission power of BTS & $-7 \mathrm{~dB}$ \\
BTS gain & $3 \mathrm{~dB}$. \\
Transmission power of the MS & $33 \mathrm{dBm}$. \\
Height of transmission antenna & $2 \mathrm{~m}$ \\
Noise RSSI & $-63 \mathrm{~dB}$ \\
\hline
\end{tabular}

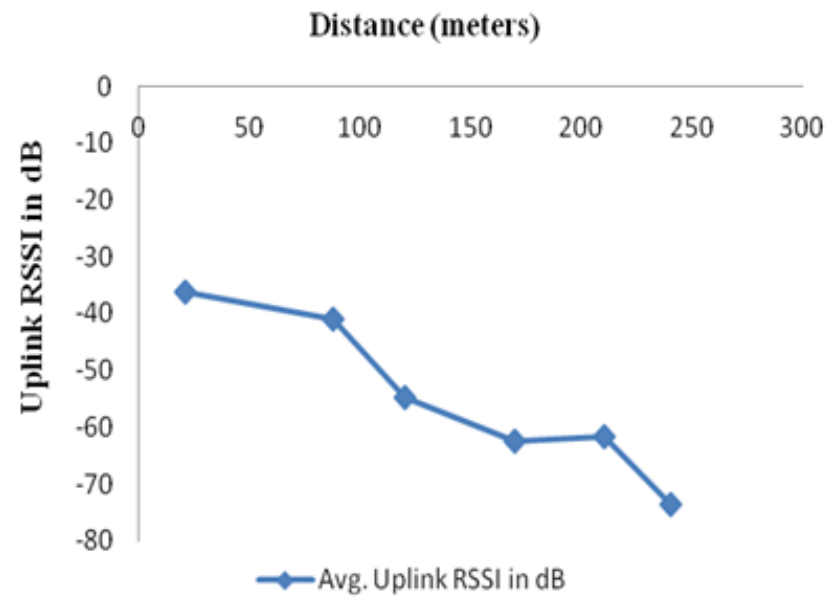

Fig. 3. Uplink RSSI $(d B)$ against Distance $(m)$

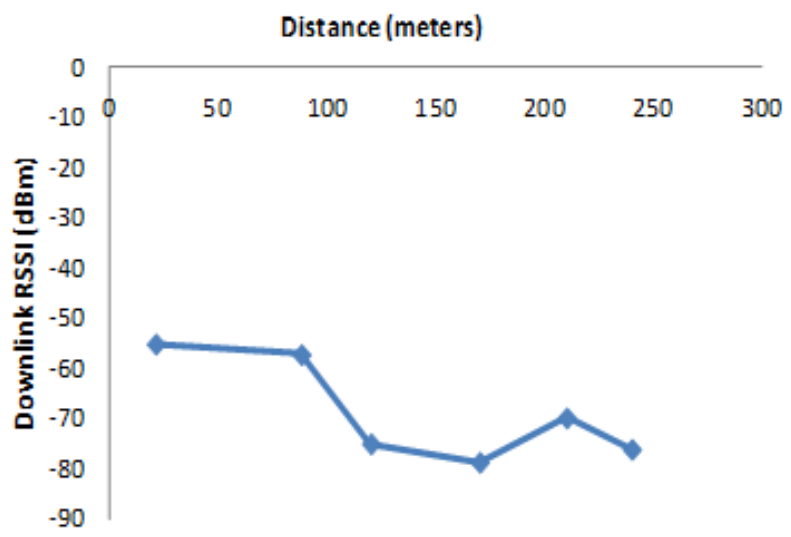

-Avg. Downlink RSSI in dBm

Fig. 4. Downlik RSSI $(d B m)$ against Distance $(m)$

distances. The results show that as the distance of separation between MS and BTS increases the downlink RSSI decreases. This means that there is more path loss as MS moves away from BTS.

To observe the variation of uplink frame erasure rate with distance, values of uplink FER were calculated at different distances. Figure 5 illustrates the testing results where percentage of frame erasure rate increases with distance due to free space loss and other attenuations which are function of distance. Apart from distance of separation between MS and BTS, transmission channel noise, interference, distortion and wireless multipath fading contribute on increase of FER. These factors are therefore cause the non-uniform

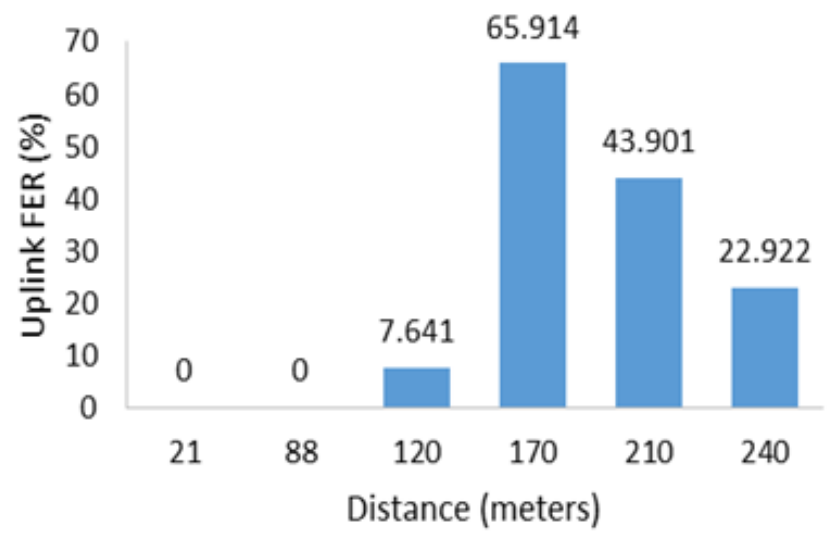

Fig. 5. Uplink FER (\%) against Distance $(m)$

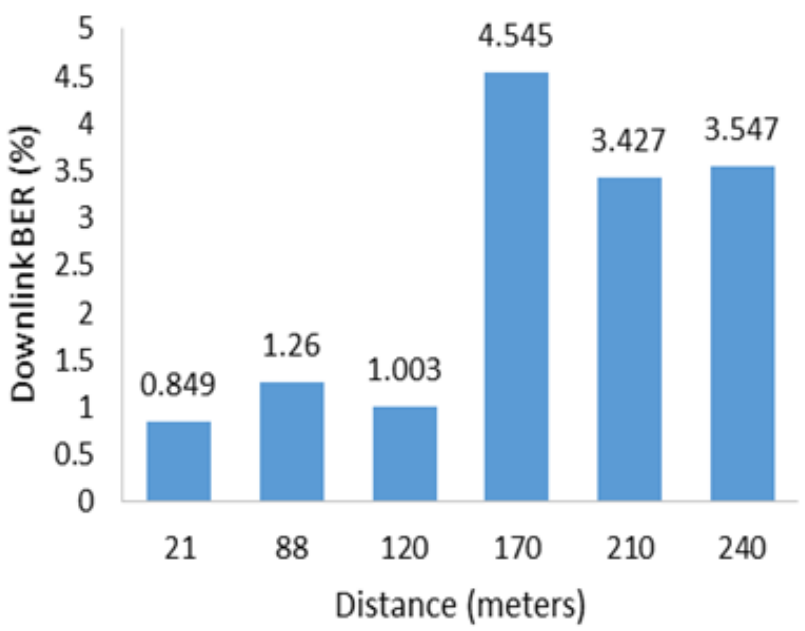

Fig. 6. Downlink BER (\%) against Distance $(m)$

nature of the graph. Figure 6 illustrate the variation of downlink BER with distance, it can be observed that percentage of BER increases with the increase in distance of separation between MS and BTS due to free space loss and other attenuation which are function of distance. Increase of BER is also caused by transmission channel noise, interference, distortion, bit synchronization problems and wireless multipath fading hence results in non-uniformity of the graph. The testing results show that the maximum network coverage with high preserved voice quality of the signal is 250 meters. Beyond this distance the voice quality is poor and unrecognizable

\section{CONCLUSION}

The prototype for a low cost cellular network was well designed and evaluated for use in rural areas based on cellular open source technology. OpenBTS along with USRP, GNU Radio and Asterisk were chosen for design and set up network for mobile communication between mobile station and Base Transceiver Stations. The prototype enabled subscribers to send and receive calls and SMS in a private single-cell GSM network in a laboratory setting. The prototype was then tested to obtain maximum network coverage and variation of various in-call parameters with distance. Testing re- 
sults revealed that Uplink and Downlink RSSI decreased with distance and Uplink FER and downlink BER increased with distance. Therefore, for the best quality voice communication between subscribers a maximum distance of $250 \mathrm{~m}$ between MS and BTS was maintained. Immediate future work will focus on setting up the proposed prototype in a typical rural setting, evaluating its real time performance and consequently designing a stable, low power and cost-effective off-the shelf cellular network for rural communications in developing countries.

\section{REFERENCES}

[1] Tanzania starts USF tenders, 2012.

[2] OpenBTS 4.0 offers new opportunities for Africa's mobile operators Targeting remote locations and the transition to IP, 2014.

[3] Abhinav Anand, Veljko Pejovic, Elizabeth M. Belding, and David L. Johnson. VillageCell. Proceedings of the Fifth International Conference on Information and Communication Technologies and Development - ICTD '12, page 180, 2012.

[4] Julie Bort. Burning Man's open source cell phone system could help save the world, 2010.

[5] Open Bts and Matt Mallett. Wireless Security Lab and OpenBTS. Technical report, Iowa State University, 2013.

[6] Communication Engineering. International Journal of Electronics and (c) I a E M E. 4(1):18-24, 2013.

[7] Michael AdeyeyeEmail author Gardner-Stephen and Paul. The Village Telco project: a reliable and practical wireless mesh telephony infrastructure. EURASIP Journal on Wireless Communications and Networking, (DOI: 10.1186/16871499-2011-78).

[8] Saim Ghafoor, Kenneth N Brown, and Cormac J Sreenan. Experimental Evaluation of a Software Defined Radio-based Prototype for a Disaster Response Cellular Network. In International Conference on Information and Communication Technologies for Disaster Management (ICT-DM), 2015.

[9] Islam E Hashem, Ahmed Elshaarany, Ahmed Y Abdelbarr, Eslam S Hassan, and Magdi Fikri. Implementing an Enhanced Base Station using the Software Defined Radio (CUOPENBTS). In International Conference on Advances in Computational Tools for Engineering Applications (ACTEA), pages 39-42, 2012.

[10] David Burgess Samra and Harvind. The Open BTS Project. Technical report, 2008.

[11] Kunal Sankhe, Chandan Pradhan, Sumit Kumar, and Garimella Rama Murthy. Cost Effective Restoration of Wireless Connectivity in Disaster Hit Areas using OpenBTS. In Annual IEEE India Conference (INDICON), volume 210, 2014.

[12] L. C. Tran, D. T. Nguyen, F. Safaei, and P. J. Vial. An experimental study of ofdm in software defined radio systems using gnu platform and usrp2 devices. In 2014 International Conference on Advanced Technologies for Communications (ATC 2014), pages 657-662, Oct 2014.

[13] Thomas Tsou, Thomas Cooper, Robert McGwier, T. Charles Clancy, and Jeffrey Reed. Development of an open-source GSM femtocell and integrated core infrastructure. In Proceedings - IEEE Military Communications Conference MILCOM, 2012
[14] Khyati Vachhani and Rao Arvind Mallari. Experimental study on wide band FM receiver using GNURadio and RTL-SDR. In 2015 International Conference on Advances in Computing, Communications and Informatics, ICACCI 2015, pages 1810-1814, 2015.

[15] Asif Ali Zamzami, Ega Putra Devara, Joni Pramana, Amang Sudarsono, and Ahmad Zainudin. Reliability Analysis of GSM Network Using Software Defined Radio-Based System. In International Electronics Symposium (IES), pages 274279, 2015. 